Cahiers $d u$ MONDE RUSSE

\section{Cahiers du monde russe}

Russie - Empire russe - Union soviétique et États indépendants

$51 / 1 \mid 2010$

Pierre le Grand et ses images de Rome

\title{
Les guides de la Rome antique dans la bibliothèque de Pierre le Grand
}

Guides of ancient Rome from Peter the Great's library

\section{Philippe Sénéchal}

\section{(2) OpenEdition}

Journals

Édition électronique

URL : https://journals.openedition.org/monderusse/9174

DOI : 10.4000/monderusse. 9174

ISSN : $1777-5388$

Éditeur

Éditions de l'EHESS

Édition imprimée

Date de publication : 15 avril 2010

Pagination : 87-100

ISBN : 978-2-7132-2306-8

ISSN : $1252-6576$

Référence électronique

Philippe Sénéchal, « Les guides de la Rome antique dans la bibliothèque de Pierre le Grand », Cahiers du monde russe [En ligne], 51/1 | 2010, mis en ligne le 10 mai 2013, consulté le 03 septembre 2022.

URL : http://journals.openedition.org/monderusse/9174; DOI : https://doi.org/10.4000/monderusse. 9174

Ce document a été généré automatiquement le 3 septembre 2022.

Tous droits réservés 


\title{
Les guides de la Rome antique dans la bibliothèque de Pierre le Grand
}

\author{
Guides of ancient Rome from Peter the Great's library
}

Philippe Sénéchal

1 L'élaboration d'un nouveau catalogue de la bibliothèque de Pierre le Grand permet de jeter un regard neuf et plus précis sur les outils dont le tsar disposait pour façonner son image de la Rome antique et élaborer sa conception d'une ville-phare pour son empire. Un point particulier et relativement secondaire à première vue, le repérage des guides de l'Urbs, offre une piste intéressante pour comprendre une partie fondamentale de l'outillage intellectuel dont s'était muni le souverain russe. Avec toutes les précautions nécessaires pour évaluer la consistance d'un fonds qui a connu de nombreux aléas, il est tout de même possible de faire des considérations sur le choix des titres retenus par Pierre et ses conseillers en matière de livres, comme Johann Daniel Schumacher. Le recollement et l'examen livre à livre ont permis des corrections importantes par rapport à l'inventaire publié en $1978^{1}$, en particulier grâce au travail mené sous l'égide d'olga Medvedkova. Il a ainsi été possible de radier de l'inventaire de la bibliothèque de Pierre le Grand certains volumes de la bibliothèque de l'Académie des sciences qui, en fait, ne provenaient pas du fonds pétrovien initial. C'est le cas pour l'exemplaire de Roma antica e moderna publié à Rome chez Giovanni Domenico Franzoni en 1653, qui venait de la collection du comte Gersdorff à Bayreuth ${ }^{2}$, ou la deuxième édition de Roma vetus ac recens d'Alessandro Donati, parue à Rome en 1639, chez Manelfo Manelfi, volume qui ne porte pas l'estampille pétrovienne ${ }^{3}$. Cependant, trois titres importants qui ont fait partie de la bibliothèque de la Chambre des apothicaires de Moscou et qui furent apportés à Saint-Pétersbourg par Pierre le Grand, n'ont, jusqu'ici, pas été retrouvés : il s'agit d'Urbis Romae Topographia de Bartolomeo Marliano (ou Marliani) ${ }^{4}$, publié à Rome par Valerio et Luigi Dorico en 1544, qui est la troisième édition de ce traité illustré, d'Urbis Romae Sciographia ex antiquis Monumentis accuratiss. delineata d'Étienne Dupérac de 1574, qui est le second grand plan de la Rome antique gravé par l'antiquaire et architecte français, et d'Antiquae Urbis splendor de Giacomo Lauro, dans son édition de $1612^{5}$. 
2 Le traité de Marliano marque une étape décisive dans les publications sur la Rome antique, d'une part grâce à ses trois plans xylographiés qui évoquent l'évolution des enceintes jusqu'à Aurélien [ill. 1] en se fondant sur des enquêtes de terrain approfondies, d'autre part grâce à ses trois reproductions gravées de statues antiques (la Louve avec Romulus et Rémus, Laocoon et l'Hercule du Capitole), les premières d'un genre appelé à une immense fortune. La plupart des éléments d'architecture en revanche sont des repiquages des gravures du traité de Sebastiano Serlio.

1

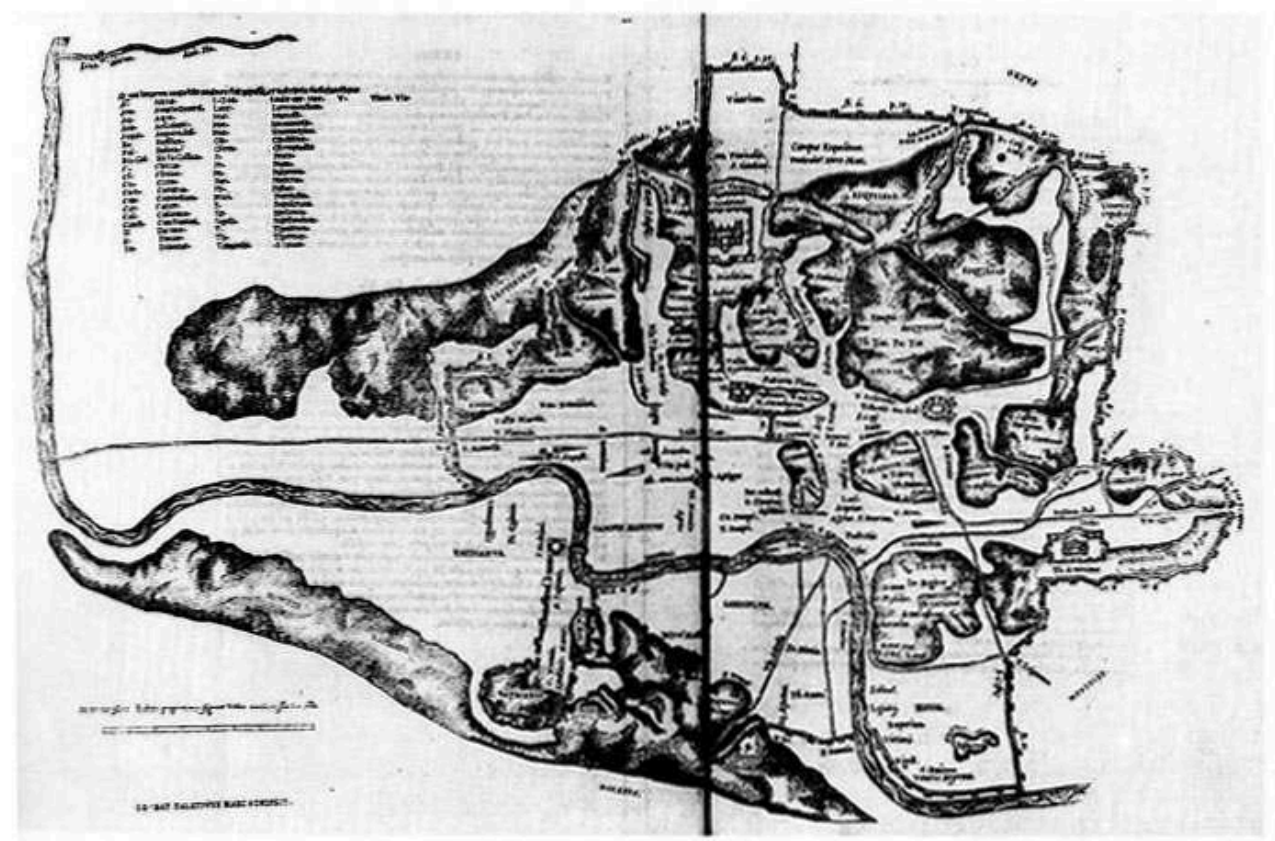

Plan de Rome avec la muraille d'Aurélien, dans Bartolomeo Marliano, Urbis Romae Topographia, Rome Valerius \& Aloysius Doricus, 1544, burin. [D.R.]

Le plan d'Étienne Dupérac (vers 1525-1585) que détint Pierre le Grand n'était pas celui de l'édition originale publiée par Lorenzo Vaccari en 1575 mais le troisième tirage, celui de l'éditeur Giovanni Giacomo De' Rossi, qui faisait suite à celui de Francesco Villamena. Il s'agit d'une impression assez tardive si nous considérons les dates de De' Rossi (1627-1691). Dans ce plan, constitué de huit planches de grandes dimensions, Dupérac fait une tentative magnifique et ambitieuse de reconstitution des bâtiments antiques, qui tient souvent de l'extrapolation hasardeuse, mais au pouvoir de suggestion grandiose [ill. 2]. La densité et la pompe de l'Urbs ne pouvaient pas ne pas frapper l'empereurbâtisseur qu'était Pierre I Ir. Mais ce dernier ne possédait pas le chef-d'œuvre de Dupérac, I Vestigi dell'antichità di Roma raccolti et ritratti in perspettiva, publié par Lorenzo Vaccari en 1575 , et qui témoigne d'une attention fidèle aux ruines ${ }^{6}$. 


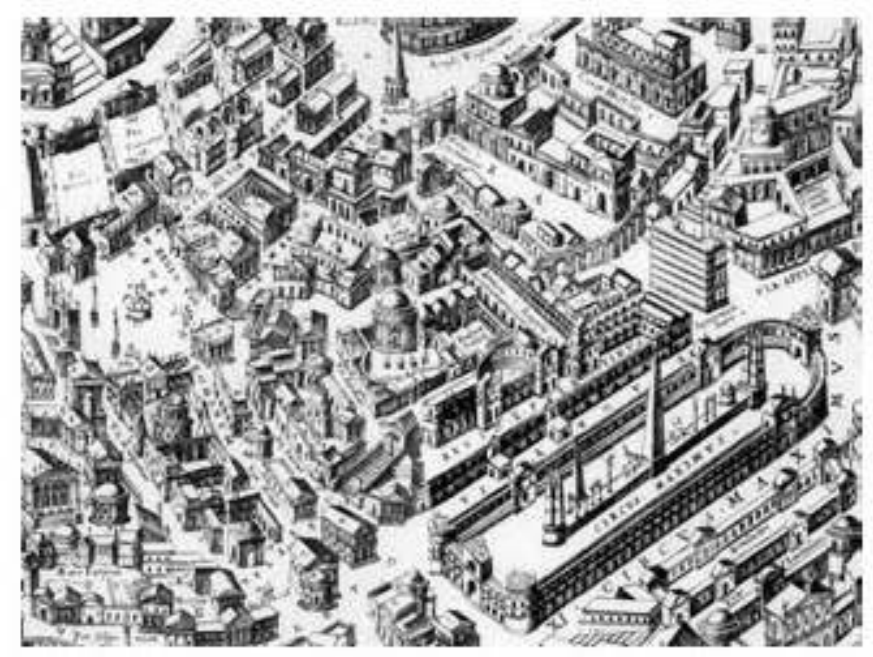

Étienne Dupérac, Vue du Palatin et du Cirque Maxime, détail de Urbis Romae Sciographia ex antiquis Monumentis accuratiss. delineata, 1574, burin. [D.R.]

4 Nous ignorons quelle édition d'Antiquae Urbis splendor fit partie de la bibliothèque du tsar mais nous savons que Giacomo Lauro (v. 1561-1645/1650), à la fois l'auteur des planches et l'éditeur, commença la gravure au burin de ces estampes et leur description vers $1586^{7}$ et travailla vingt-huit ans à ce qui est considéré comme son opus magnum. L'ouvrage évoque, dans ses gravures sur cuivre assorties de commentaires, les principaux sites et édifices de la Ville éternelle [ill. 3], et fait suivre les estampes, d'une qualité modeste, d'un bref traité historique sur Rome et ses grands personnages, des rois aux empereurs. Cet ouvrage fut l'un des plus diffusés du XvII siècle.

3

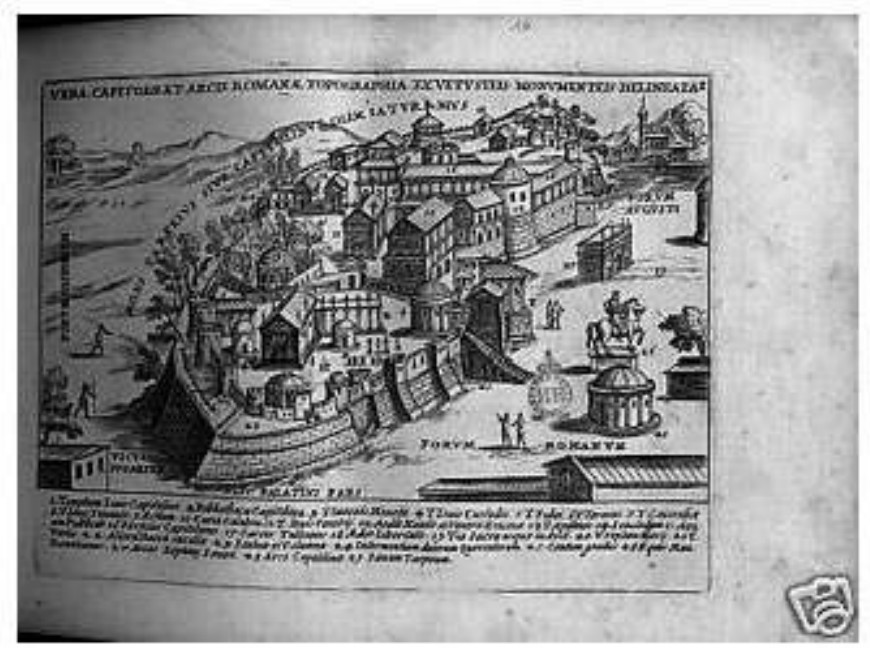

Le Capitole, dans Giacomo Lauro, Antiquae Urbis splendor, s. I., 1612, burin. [D.R.]

5 Pour ce qui est de la production romaine des $\mathrm{XVI}^{\mathrm{e}}$ et $\mathrm{XVII}{ }^{\mathrm{e}}$ siècles, Pierre le Grand n'avait donc à sa disposition que trois exemples, ce qui est peu, mais bien choisis: deux ouvrages unanimement appréciés et une carte impressionnante. Toutefois, rappelons-le, ces documents n'ont toujours pas été mis au jour. Dans la bibliothèque de l'Académie des 
sciences, la récolte n'est pas beaucoup plus abondante, ce qui est tout à fait révélateur. Dans le strict champ des guides de la Rome antique, nous n'avons retrouvé que trois titres.

6 Avant même d'examiner ces livres, nous voudrions signaler deux absences frappantes. Notons tout d'abord celle de grands classiques de la topographie antique. Si l'on fait abstraction des trois volumes publiés à Rome qui faisaient partie du fonds des apothicaires moscovites, manquent en effet à l'appel la plupart des antiquaires topographes des $\mathrm{Xv}^{\mathrm{e}}$ et $\mathrm{xVI}^{\mathrm{e}}$ siècles, tant les Italiens Francesco Albertini, Flavio Biondo, Pomponio Leto, Marco Fulvio Calvo, Andrea Fulvio, Lucio Fauno, Bernardo Gamucci ou Pirro Ligorio, que le Français Jean-Jacques Boissard ou l'Allemand Georg Fabricius von Chemnitz, sans parler de moult autres guides moins ambitieux à destination des visiteurs et des pèlerins, cités dans le livre fondamental de Ludwig Schudt, Le Guide di Roma ${ }^{8}$. Pierre le Grand et Schumacher n'ont donc pas jugé nécessaire de faire une collecte systématique de la littérature des érudits de la Renaissance, alors même que ces écrits furent la base de toutes les compilations successives. Une telle collecte aurait certes demandé une recherche auprès de plusieurs libraires ou agents en Europe mais, pour des auteurs aussi fameux, elle n'aurait pas été si difficile.

7 En second lieu, la bibliothèque de Pierre le Grand ne comprenait pas non plus les compendiums fondamentaux publiés dans les Provinces-Unies alors même que le tsar y faisait son premier séjour. Il s'agit notamment du Thesaurus Antiquitatum Romanarum de Johann Georg Graevius, publié en 12 volumes in-folio à Utrecht et à Leyde de 1694 à 1699, et du Thesaurus Graecarum Antiquatum de Jacob Gronovius, paru à Leyde en 13 volumes in-folio de 1697 à 1702, ouvrages qui reprennent la plupart des textes des érudits antérieurs ${ }^{9}$. L'acquisition de ces recueils volumineux mais commodes aurait dispensé les pourvoyeurs des recherches des volumes originaux des antiquaires de la Renaissance et du XVII ${ }^{e}$ siècle. Et pourtant, l'un des coéditeurs de ces sommes érudites, Pieter van der Aa, était en correspondance régulière avec Johann Daniel Schumacher ${ }^{10}$. Le 25 avril 1724, l'éditeur leydois avait en outre suggéré à ce dernier d'acquérir les planches de cuivre qui avaient servi aux Thesauri, au cas où l'empereur aurait voulu créer une "Chambre des Tableaux de cuivre, mis dans des quadres avec verre devant». Mais ce cabinet, "qui n'aura[it] pas eu son pareil dans le monde », ne vit pas le jour ${ }^{11}$. 


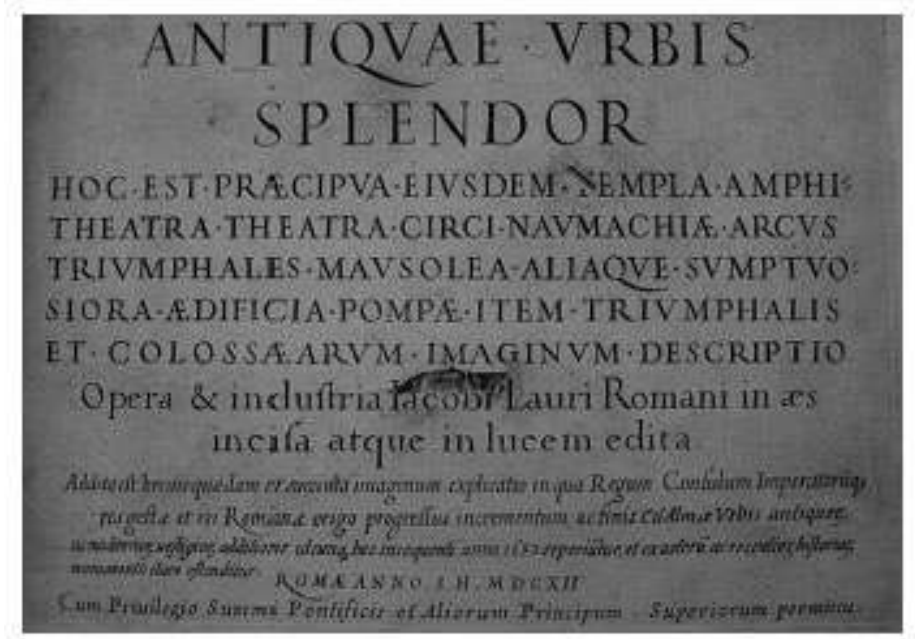

Giacomo Lauro, Antiquae Urbis splendor, s. I., 1637, page de titre. [D.R.]

8 Il semblerait donc que la nécessité de s'entourer du corpus des autorités sur la topographie de la Rome antique n'ait pas été sentie comme primordiale par le tsar. Nous pouvons dès lors hasarder deux hypothèses: d'abord, si certains types de bâtiments, éléments symboliques, ou motifs narratifs prestigieux entraient aisément dans un dessein de création d'une nouvelle ville impériale à l'époque moderne, d'autres étaient parfaitement inadaptés; ensuite, la topographie de Rome, avec ses sept collines sous un ciel clément, et la disposition très particulière de ses édifices prestigieux ne sont pas facilement transposables dans une ville nordique construite sur des marais. Peut-être était-il suffisant au tsar, grâce à deux ou trois traités fondamentaux et à des guides récents et commodes, de pouvoir rapidement resituer sur un plan avec les quatorze régions de la Ville éternelle les bâtiments ou les monuments les plus fameux sans entrer dans les querelles ou les pinaillages des antiquaires sur les emplacements ou les reconstitutions. Mais il ne s'agit là que de conjectures car, si l'on en croit Paul Gerardus Hoftijzer, le grand spécialiste de Pieter van der Aa, une bonne partie des ouvrages publiés par cet éditeur et acquis pour Pierre le Grand périt dans un incendie survenu en 1988 dans la bibliothèque de l'Académie des sciences de Saint-Pétersbourg ${ }^{12}$. Qui sait donc si les Thesauri ne faisaient pas partie des ouvrages qui furent la proie des flammes?

9 De fait, les trois titres retrouvés dans le fonds pétersbourgeois ne sont pas des textes majeurs mais des manuels ou des guides illustrés qui fournissent, en allemand, en latin ou en hollandais, la doxa sur la Rome antique dans la seconde moitié du XVII siècle autour de 1700, et mentionnent tous les monuments célèbres. Dans tous les cas, ils étaient accompagnés d'un pendant pour la Rome moderne. Par ordre d'ancienneté, le premier ouvrage sur ces matières est un guide intitulé Abgebildetes Altes Romm, publié en 1662 à Arnhem par Johann Friedrich Haagen [ill. 5 $]^{13}$. Cet in-12 de 338 pages est en fait la traduction en Hochdeutsch par le magister et poète de Lübeck Albert Reimarus († 22 sept. 1641) du manuel de Pompilio Totti, Ritratto di Roma antica, dont la première édition romaine remontait à $1627^{14}$. Ce succès italien fut réimprimé en 1633 , avant d'être repris de nombreuses fois par l'éditeur Filippo De' Rossi et ses successeurs à partir de 1645. L'éditeur arnhemois a accompagné le texte de 128 figures en cuivre reproduisant les monuments clés de l'Urbs antique; ces illustrations sont de qualité assez modeste, curieusement dotées de légendes en néerlandais et non en allemand [ill. 6]. 


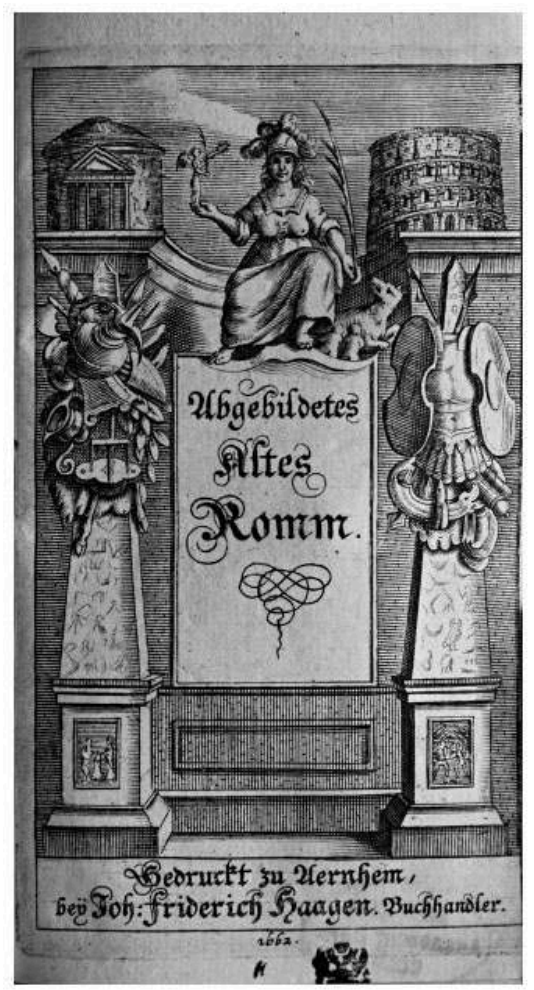

Albert Reimarus, Abgebildetes Altes Romm, Arnhem : Johann Friedrich Haagen, 1662, frontispice, SPb., BAN, P I, n P.O. 316. 


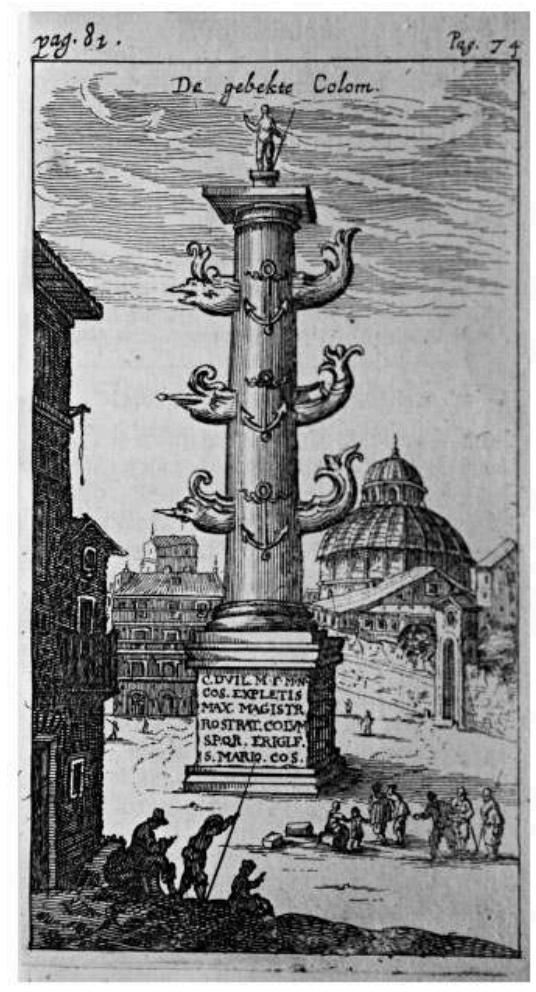

Colonne rostrale à Rome, dans Albert Reimarus, Abgebildetes Altes Romm, Arnhem : Johann Friedrich Haagen, 1662, p. 81, SPb., BAN, P I, n P.O. 316.

Le deuxième manuel est une édition néerlandaise du traité du jésuite siennois Alessandro Donati (1584-1640), Roma vetus ac recens ${ }^{15}$. Il s'agit d'une édition in- $4^{\circ}$ en 356 pages, datant de 1695 , alors que, nous l'avons déjà signalé, la première avait vu le jour en 1639 , avec une dédicace à Urbain VIII, pape qui redonnait lustre et pompe à la ville de Rome ${ }^{16}$. Une version corrigée du livre de Donati avait été publiée par le même Filippo De' Rossi en 1648, et réimprimée en 1665. La version de 1695 publiée à Amsterdam, et détenue par Pierre le Grand, est considérablement revue et augmentée, dotée d'un long index et de 98 planches gravées sur cuivre. Par rapport au frontispice de 1639, gravé par Johann Friedrich Greuter (vers 1595, Strasbourg-1662, Rome) d'après Giovanni Antonio Lelli (avant 1580, Rome-1640, Rome), celui de 1695 modifie légèrement la forme de l'allégorie de Rome assise, remplace les anges tenant des trophées antiques ou des emblèmes religieux par des aigles servant d'accoudoirs au trône, et donne plus de visibilité à la louve et aux jumeaux [ill. 7]. Les représentations des bâtiments sont un peu plus précises que dans le livre de Reimarus; elles sont accompagnées de relevés d'inscriptions, mais souvent décontextualisées [ill. 8]. Les coéditeurs de cette version néerlandaise du manuel de Donati étaient Gillis et Johannes Janssonius van Waesberge en association avec Johannes Wolters. Or, en décembre 1689, ce même Johannes Janssonius s'était plaint à son correspondant londonien, Samuel Smith, de l'état de l'édition, perturbé par les guerres avec la France: "C'est un malheur général à présent le misérable état de la librairie, laquelle ne désire que la paix [...]. On imprime fort peu à présent dans nos pais comme aussi partout $\aleph^{17}$. L'éditeur amstellodamois se serait certainement réjoui de l'ouverture d'un nouveau marché du livre grâce aux ambitions du jeune tsar. 


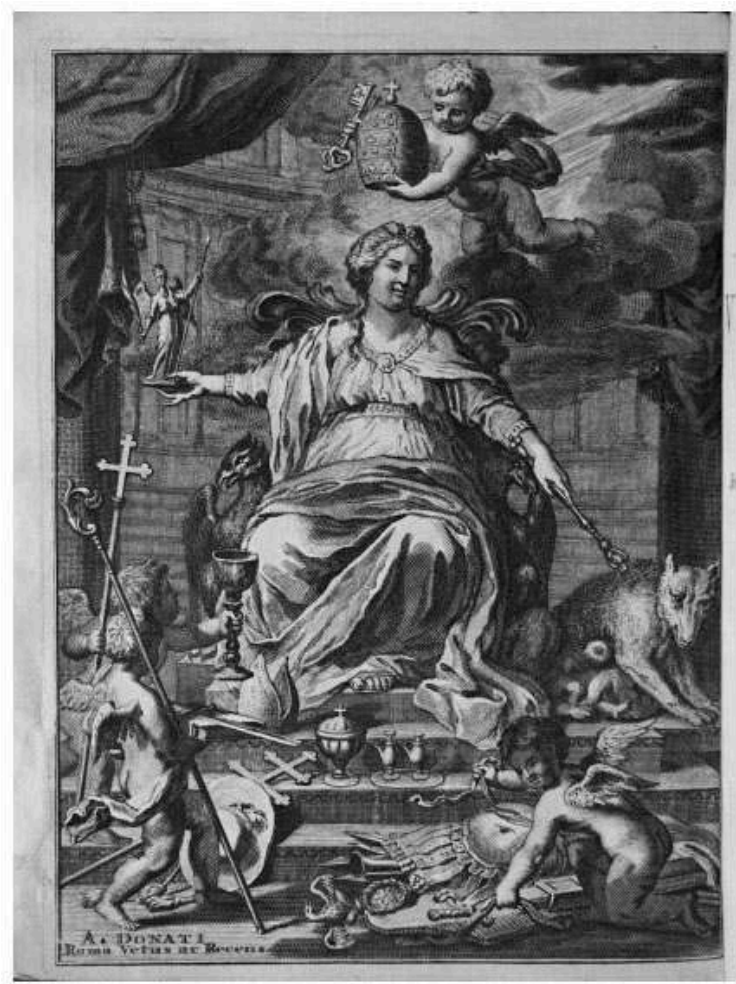

Alessandro Donati, Roma vetus ac recens, Amstelodæmi : apud Janssonio-Waessbergios \& Joannem Wolters, 1695, frontispice, burin, SPb., BAN, P I, n P.O. 254. 


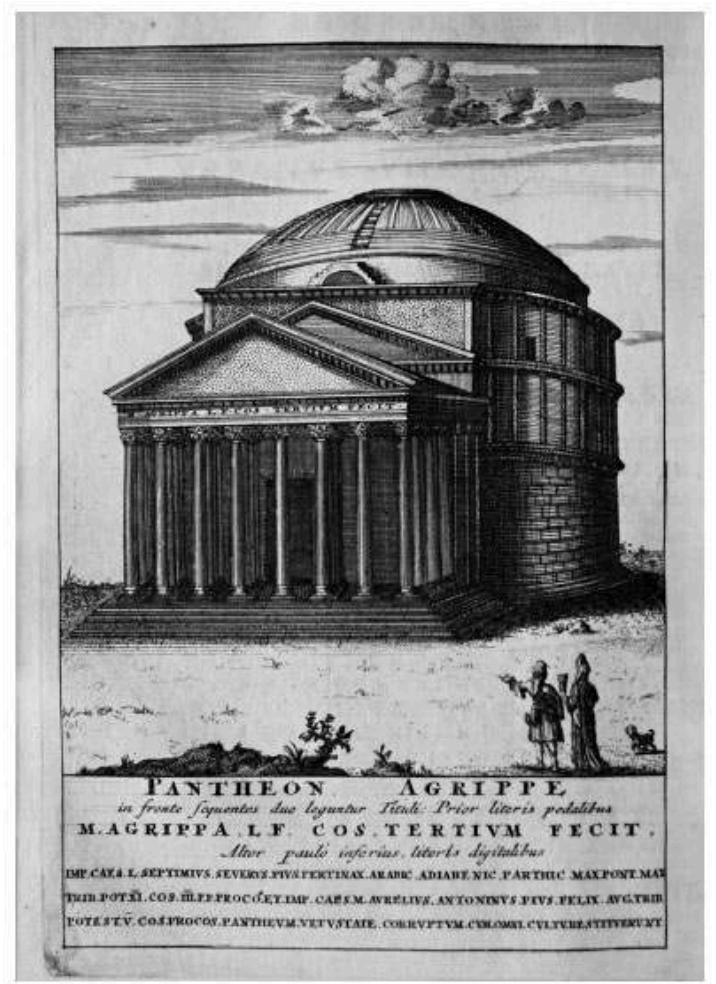

Le Panthéon d'Agrippa à Rome, dans Alessandro Donati, Roma vetus ac recens, Amstelodæmi : apud Janssonio-Waessbergios \& Joannem Wolters, 1695, burin, SPb., BAN, P I, n P.O. 254.

Le dernier titre est le plus important : il s'agit d'un in-folio de 1704, Beschryving van oud en Nieuw Rome [Description de Rome antique et moderne], conservé en deux exemplaires dans la bibliothèque de Pierre le Grand, dont un à la reliure très usée, ce qui laisse entendre un maniement fréquent [ill. 9] ${ }^{18}$. Il s'agit de la traduction néerlandaise d'un manuel dû au Français François Joseph Deseine (ou Desseine), un libraire établi à Rome $(† 1715)$. Cet érudit avait déjà publié de commodes in-12 à destination des voyageurs, Description de la ville de Rome en faveur des étrangers à Lyon chez J. Thioly en 1690 (4 vol.) et, chez le même éditeur, Nouveau voyage d'Italie, en 1699 (2 vol.). Mais son grand œuvre, l'infolio dont Pierre le Grand posséda un exemplaire, parut d'abord en néerlandais. En effet, c'est seulement en 1713 qu'une version française de ses traités, L'Ancienne Rome et Rome moderne, vit le jour sur les presses du grand éditeur leydois, Pieter van der $\mathrm{Aa}^{19}$. Beschryving Van Oud En Niew Rome, en revanche, fut édité à Amsterdam par François Halma, qui avait été le partenaire de Van der Aa pour la publication des opera magna de Graevius et Gronovius et qui ne manque pas, dans une note de son avis au lecteur, de renvoyer à la Roma antica de Famiano Nardini - non à son édition originale (Rome : per il Falco, 1666), mais à sa traduction en latin par Jacobus Tollius (1626-1696) dans le quatrième volume du Thesaurus Antiquitatum Romanarum. 


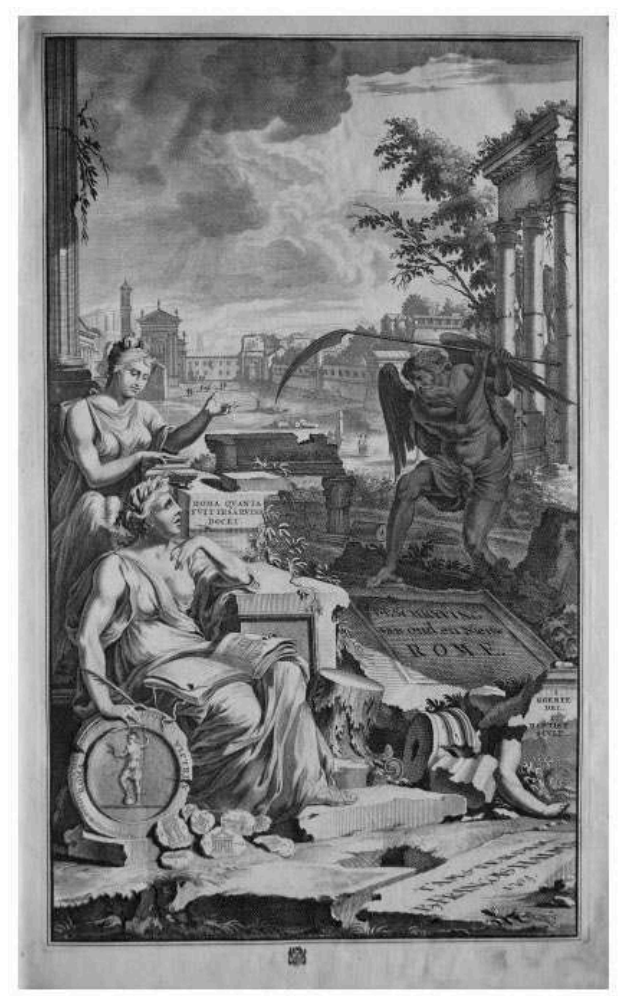

François Deseine, Beschryving van Oud en Nieuw Rome, Amsterdam : François Halma, 1704, frontispice, burin, SPb., BAN, P I, n P.O. 487.

Beschryving mérite des observations particulières car il s'agit d'un ouvrage ambitieux, complété par une réédition de la carte de l'Italie antique due au cartographe français Nicolas Sanson (1600-1667) ainsi que par une reprise des planches de Pietro Sante Bartoli assorties des commentaires de Giovan Pietro Bellori sur les peintures du tombeau des Nasons, publiés pour la première fois à Rome en 1680. Halma commanda des dessins à un peintre classicisant, sans doute disciple de Gerard de Lairesse, Jan Goeree (20 oct. 1670, Middelburg-4 janv.1731, Amsterdam), dessins qui sont en partie conservés au Metropolitan Museum de New York ${ }^{20}$, et qu'il fit graver au burin par Jacobus de Later (actif de 1696 à 1705), Lorenz Scherm (actif de 1690 environ à 1707), Peter Sluiter (1675, Amsterdam-après 1713) et Jan van Vianen (vers 1660-1726, Amsterdam). Ces estampes associent des vues des monuments à des objets antiques ayant une relation historique avec les édifices; elles combinent restitutions idéales et vues de l'époque moderne, avec des superpositions et des effets de trompe-l'œil. Ainsi, l'arc de Titus est-il montré une première fois dans son intégrité retrouvée, en haut de l'estampe et, plus bas, dans son état vers 1700, pris dans les murs de clôture du Forum romain, sur une estampe fictive en partie masquée par des monnaies de cet empereur et par une pierre gravée [ill. 10]. Le reste des planches reprend des illustrations célèbres, par exemple le plan de Rome de Pirro Ligorio ou les vues des aqueducs de Raffaello Fabretti ${ }^{21}$. On voit donc ici, en miniature, les procédés de remploi luxueux de matériaux antérieurs, qui font l'utilité et les limites des Thesauri de Gronovius et Graevius, du même éditeur. 


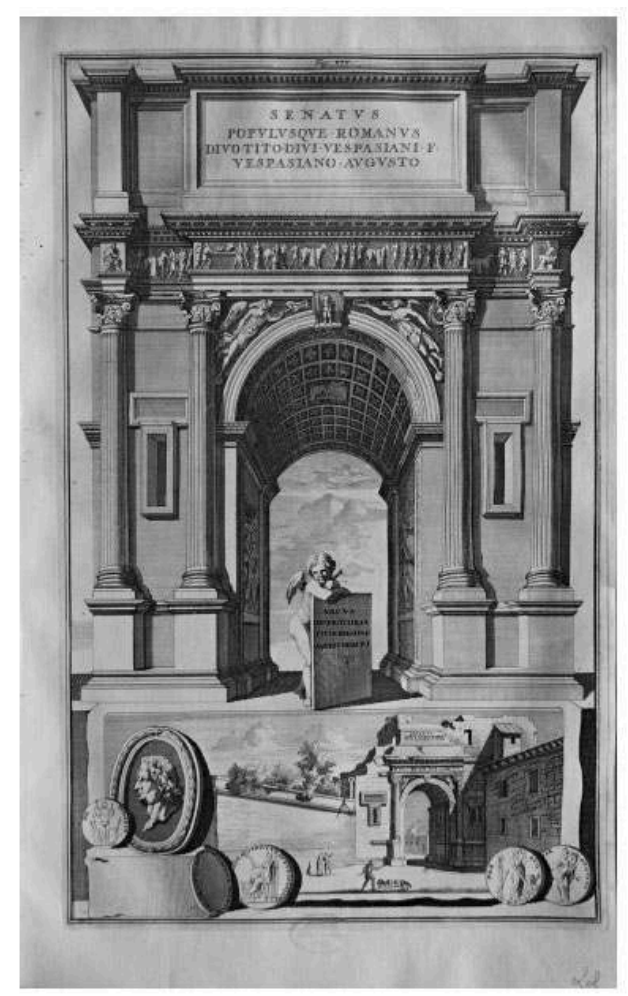

L'arc de Titus à Rome, dans François Deseine, Beschryving van Oud en Nieuw Rome, Amsterdam: François Halma, 1704, entre p. 42 et 43, burin, SPb., BAN, P I, n P.O. 487.

plaire de Beschryving a pu parvenir jusqu au tsar par lintermédiaire de son grand ami, le diplomate, cartographe, collectionneur et écrivain maritime amstellodamois Nicolaes Witsen (8 mai 1641-10 août 1717), auquel elle est dédiée. Le célèbre bourgmestre d'Amsterdam avait organisé le séjour du souverain en 1697 et devait mourir en présence du tsar lors du second voyage de ce dernier, en $1717^{22}$. Dans sa jeunesse, en 1666-1667, Witsen avait visité Rome et était véritablement en mesure d'apprécier la qualité du travail éditorial accompli par François Halma.

Enfin, une fois entré dans les collections de l'Académie des sciences, l'ouvrage de Deseine a dû beaucoup plaire aux artistes pétersbourgeois. Nous y trouvons en particulier une vue spectaculaire de la colonne Antonine à travers un arc de triomphe [ill.11] ${ }^{23}$. Cette mise en scène, qui ne correspond absolument pas à la réalité du site romain, théâtralise le monument. En outre, dans les deux exemplaires de Beschryving, les planches représentant la colonne Trajane diffèrent; dans le volume en meilleur état, la colonne, avec une légende en néerlandais, PRAALZUIL VAN TRAJANI, est présentée avec le même procédé d'encadrement théâtral [ill. 12] ${ }^{24}$. Ces deux estampes ont fort bien pu marquer, un siècle plus tard, Karl Ivanovič Rossi quand il a suggéré l'emplacement de la colonne d'Alexandre, due à Auguste Ricard de Montferrand, colonne qui se dresse depuis 1834 sur la place du palais d'Hiver. En 1819-1829, Rossi avait conçu la double arche de l'étatmajor, qui débouche de la rue Millionnaja-Longovaja, devenue plus tard Bolchaja Morskaja, offrant un encadrement grandiose à la colonne de bronze [ill. 13]. 


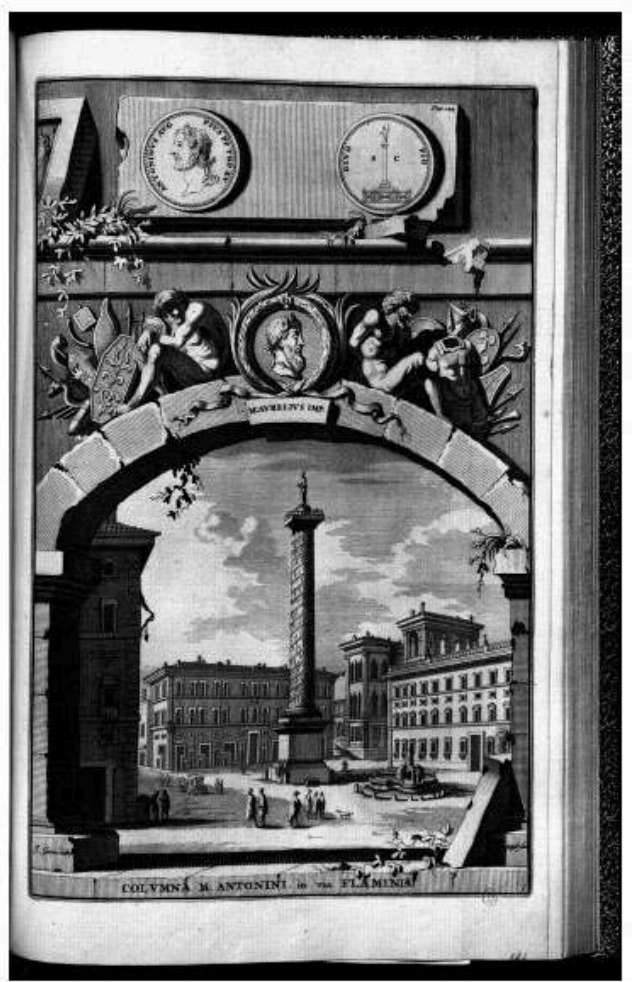

La colonne Antonine, dans François Deseine, Beschryving van Oud en Nieuw Rome, Amsterdam : François Halma, 1704, entre p. 90 et 91, burin, SPb., BAN, P I, n P.O. 487. 
12

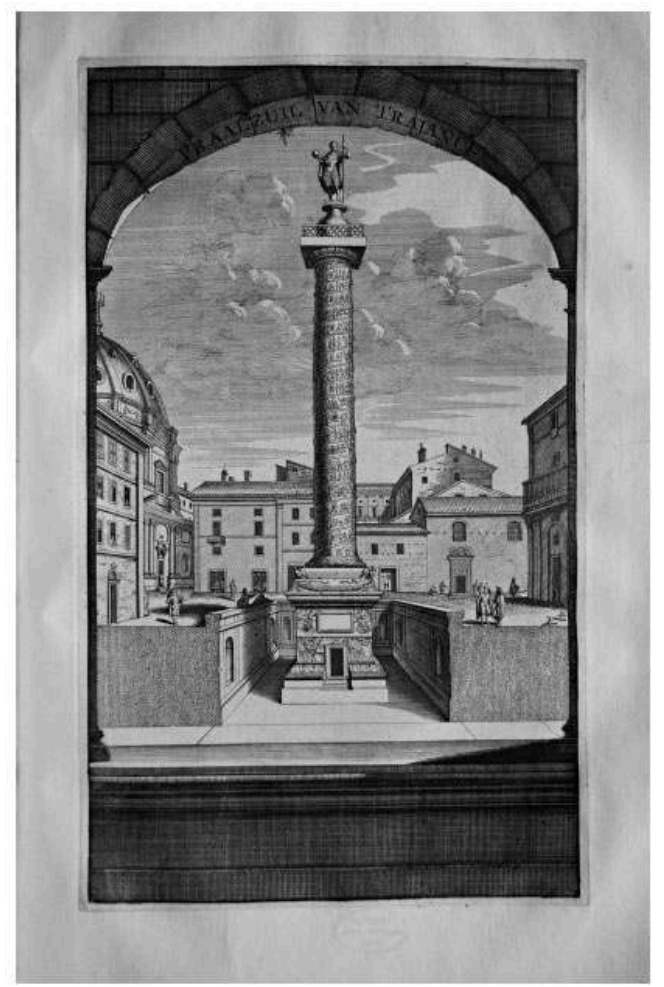

La colonne Trajane, dans François Deseine, Beschryving van Oud en Nieuw Rome, Amsterdam : François Halma, 1704, entre p. 70 et 71, SPb., BAN, n P I 487. 
13

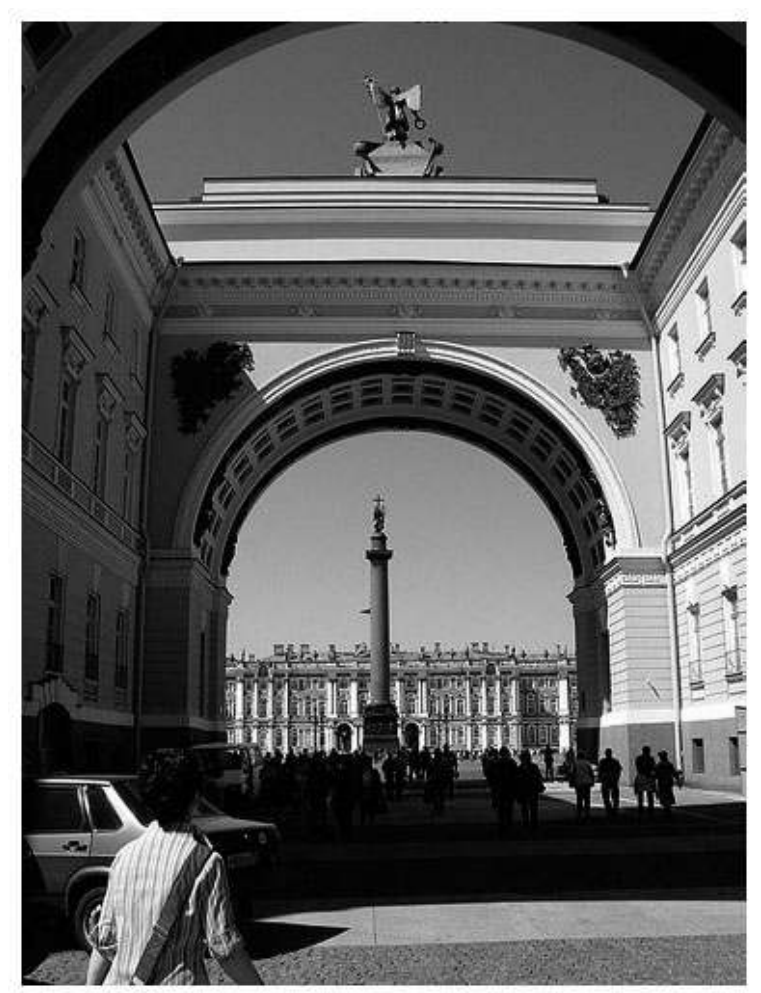

La colonne Alexandre à Saint-Pétersbourg, vue à travers l'arc de l'État-Major, depuis la rue Bol'šaja Morskaja. [D.R.]

Les trois guides de la Rome monumentale antique présents encore aujourd'hui dans la bibliothèque de Pierre le Grand ont donc été publiés dans les Provinces-Unies entre 1662 et 1704. Si les deux manuels dérivés de publications romaines sont de simples vademecum, il n'en va pas de même du livre de Deseine, d'un accès facile pour le tsar car publié en néerlandais, et qui offrait à ce dernier des planches de grande qualité, lui permettant d'avoir une vision plausible et flatteuse de la Rome antique, d'où pouvait naître une émulation, sans qu'une trop grande érudition fût nécessaire pour l'apprécier. Bien plus tard, une fois mises à la disposition des architectes et décorateurs pétersbourgeois, ces visions grandioses purent s'incarner de nouveau. On passa du musée de papier au rêve de pierre.

\section{NOTES}

1. E.I. Bobrova, Biblioteka Petra I: Ukazatel'-spravočnik [ La bibliothèque de Pierre I ${ }^{\text {er }}$ : Catalogue], L. : BAN, 1978.

2. Ibid., p. $146, \mathrm{n}^{\circ} 1444$.

3. Ibid., p. 122, n 1089. L'editio princeps avait été publiée par Manelfi l'année précédente. Sur le livre I dans l'édition de 1638, voir Colin Dale Pilney, Alessandro Donati's «Roma vetus 
ac recens", Book One; Text, translation and commentary, (January 1, 2001), ETD Collection for Fordham University. Paper AAI9999831. http://fordham.bepress.com/dissertations/ AAI9999831000 (consulté le 28 octobre 2008).

4. Bobrova, Biblioteka Petra I, p. 136, nº 1294.

5. Je remercie vivement Olga Medvedkova d'avoir effectué ces vérifications pour moi dans E.A.Savel'eva, Katalog knig iz sobraniia Aptekarskogo prikaza [Catalogue de la bibliothèque de la Chambre des apothicaires], SPb. : BAN, 2006.

6. Sur Dupérac, voir désormais Étienne Lurin, « Étienne Dupérac vedutista e cartografo: la costruzione della pianta di Roma del $1577 »$ in Cesare de Seta, Brigitte Marin, éds., avec la coll. de Marco Iuliano, Le città dei cartografi: studi e ricerche di storia urbana, Naples: Electa, 2008, p. 49-59; «Un homme entre deux mondes: Étienne Dupérac, peintre, graveur et architecte en Italie et en France (1535?-1604) », in Henri Zerner, Marc Bayard, éds., Renaissance en France, Renaissance française?, P. : Somogy Éditions d'art, 2009, p. 37-59. Sur Vaccari, ou le Français Laurent de La Vacherie, dit aussi Lorenzo della Vaccaria, voir Gian Ludovico Masetti Zannini, "Rivalità e lavoro di incisori nelle botteghe LafréryDuchet et de La Vacherie ", Les fondations nationales dans la Rome pontificale: Rome, mai 1978 / Académie de France; École française de Rome, Turin: La Bottega d'Erasmo, 1981, p. 545-566.

7. Antiquae Urbis splendor, hoc est praecipua ejudesm templa, amphitheatra, circi... aliaque sumptuosiora aedificia... opera et indiustria Jacobi Lauri... in aes incisa atque in lucem edita. Addita est brevis quaedam... imaginum explicatio, in qua regnum, consulum imperatorumque res gestae et rei romanae origo, progressus, incrementum ac finis ex historiarum monumentis ostenditur, [s. 1.], 1612. L'édition de 1612 fut souvent réimprimée avec l'insertion de planches postérieures ou la suppression de certaines planches (voir les volumes publiés en 1613 et 1615). On trouve même des éditions datées de 1612 qui annoncent dans leur titre qu'elles contiennent des références à des monuments observés jusqu'en 1630 [ill. 4]... Impossible, hélas, de savoir laquelle fit partie de la bibliothèque du tsar. Aux trois premiers livres montrant des reconstitutions de la Rome antique et des scènes évoquant les cérémonies et les coutumes fut ajouté en 1628 un quatrième représentant les monuments dans leur aspect actuel et des vues de la Rome moderne. D'autres éditions suivirent, en 1637 et 1641. Dans sa forme finale, Antiquae Urbis splendor comprend 167 planches. Sur Giacomo Lauro, voir Thomas Ashby, «Un incisore antiquario del Seicento ", La Bibliofilia, 27, 1926-1927, p. 361-373 ; 28, 1927, p. 453-460 ; 29, 1927-1928, p. 356-369, XXXI, 1929, p. 105-122 ; Michael Bury, The Print in Italy 1550-1620, Londres: The British Museum Press, 2001, p. 126-128, 134, note 140, et 228, avec bibl. ant.

8. Ludwig Schudt, Le guide di Roma: Materialien zu einer Geschichte der römischen Topographie: Unter Benützung des handschriftlichen Nachlasses von Oskar Pollak, Vienne et al. : Filser, 1930. On se reportera aussi au remarquable ouvage de Margaret Daly Davis, Archäologie der Antike : aus den Beständen der Herzog August Bibliothek 1500-1700, cat. expo. (Wolfenbüttel, 16 juil.-2 oct. 1994), Wiesbaden : Harrassowitz in Komm.

9. Joannes Georgius Graevius, Thesaurus Antiquitatum Romanarum, Trajecti ad Rhenum : Apud Franciscum Halmam ; Lugduni Batavorum : Petrum vander Aa, 1694-1699; Jacobus Gronovius, Thesaurus Graecarum Antiquitatum, Lugduni Batavorum : Petrus \& Baldinus Vander Aa, 1697-1702. Sur ces grandes entreprises d'érudition, voir Paul Gerardus Hoftijzer, Pieter van der Aa (1659-1733): Leids drukker en boekverkoper, Hilversum: Verloren, 1999, p. 33-34, 36-41, 68, 71-72. 
10. N.A. Kopanew, «"Gollandskij” izdatel' P'er van der Aa.: Biblioteke Petersburgskoj Akademii Nauk [L'éditeur hollandais Pieter van der Aa à la bibliothèque de l'Académie des sciences de Saint-Pétersbourg] », in A.A. Zajceva et al., éd., Kniga $v$ Rossii XVIII-serediny XIX v. iz istorii Biblioteki Akademii Nauk [Le livre dans la Russie du XVIII et de la première moitié du XIXe siècle], L. : Biblioteka Akademii Nauk CCCP, 1989, p. 42-51; P.G. Hoftijzer, «The Leiden bookseller Pieter van der Aa (1659-1733) and the international book trade » in Christiane Berkvens-Stevelinck et al., éd., Le Magasin de l'Univers. The Dutch Republic as the centre of the European book trade: papers presented at the international colloquium, held at Wassenaar, 5-7 July 1990, Leyde et al. : E.J. Brill, 1992, p. 169-184.

11. Cité dans Kopanew, «“Gollandskij” izdatel'... », p. 49.

12. Hoftijzer, Pieter van der Aa, p. 84.

13. SPb, BAN OR P I. (Otdel Rukopisej Biblioteka Akademii Nauk P I - Département des Manuscrits, Bibliothèque de l'Académie des sciences, Pierre $\left.\mathrm{I}^{\mathrm{er}}\right), \mathrm{n}^{\circ}$ inv. $316 \mathrm{P} \mathrm{I}$ in.

14. Pompilio Totti, Ritratto di Roma antica: nel quale sono figurati i principali tempij, teatri, anfiteatri, cerchi, naumachie, archi trionfali, curie, basiliche, colonne, ordine del trionfo, dignità militari, e civili, riti, cerimonie, medaglie \& altre cose notabili; con le vite, \& effigie de' primi re d'essa. E le dichiarationi di Bartolomeo Marliani, e d'altri autori, Roma : Per Andrea Fei, 1627.

15. SPb. : BAN OR P I, $n^{\circ}$ P.O. inv. 254 P I in.

16. Alexandri Donati e Societate Jesu Roma vetus ac recens Utriusque Ædificiis illustrata. In multis locis aucta, castigior reddita, indice locupletißimo, et Figuris Æneis illustrata, Editio Ultima, Amstelodæmi : apud Janssonio-Waessbergios \& Joannem Wolters, 1695.

17. Lettre du 16 décembre 1689, Oxford, Bodleian Library, correspondance de Samuel Smith, Rawlinson MSS, Letters 114, f. 188; cité dans Hoftijzer, «Het nederlandse boekenbedrijf omstreeks 1700 ", in Renée Kistemaker, Natalja Kopaneva, Annemiek Overbeek, éds., Pieter de Grote en Holland: Culturele en wetenschappelijke betrekkingen tussen Rusland en Nederland ten tijde van tsar Pieter de Grote, cat. expo. (Amsterdam, Historisch Museum, 17 déc. 1996-13 avr. 1997), Amsterdam : Historisch Museum, et Bussum : Uitgeverij THOTH, 1996, p. 103-107, p. 107, note 6.

18. SPb.: BAN OR P I, n P.O. inv. 472 P I in. (le plus usé); et n' P.O. inv. 487 P I in. En outre, Beschryving Van Oud En Niew Rome, du même Deseine, et publiée aussi séparément la même année par le même éditeur, comme en témoigne un autre exemplaire conservé dans la bibliothèque de Pierre le Grand, comprend aussi parfois des vues d'édifices antiques, souvent d'après des dessins du Gantois établi à Rome Lieven Cruyl (vers 1640vers 1720). Les deux planches sur la colonne rostrale et celle sur la colonne Trajane (pl. 9 et 10) sont identiques à celles de l'exemplaire $n^{\circ} 472$, mais avec des légendes différentes ["Columna Rostrata, de Schipsbezzigde Zuyl» et "Columna Trajani, de Zuyl van Keizer Trajanus »]. La partie sur la Rome moderne est dédiée au bourgmestre de Leyde, Coenraad Ruysch († 1731).

19. Voir Hoftijzer, Pieter van der Aa, p. 44.

20. Department of Drawings and Prints, inv. 58.648.2 (1-55), Gift of Helen and Janos Scholz.

21. Raffaello Fabretti, De aquis et aquaeductibus veteris Romae, dissertationes tres, Romae: apud typis Ioannis Baptistae Bußotti, 1680.

22. Sur les dédicaces d'ouvrages à Nicolaes Witsen, voir Marion Peters, "Nepotisme, patronage en boekopdrachten bij Nicolaes Witsen (1641-1717), burgemeester van 
Amsterdam ", Lias : sources and documents relating to the early modern history of ideas, 25, 1998, I, p. 83-134 ; http://www.xs4all.nl/ mhpeters/nep.htm (consulté février 2011). Voir aussi Jozien J. Driessen, Tsaar Peter de Grote en zijn Amsterdamse vrienden [Pieter the Great and his friends in Amsterdam], Utrecht : Kosmos, 1996.

23. Entre les p. 90 et 91 (COLUMNA M. ANTONINI in via FLAMINIA).

24. Les planches n'ont pas été reliées de façon tout à fait identique dans les deux exemplaires. En outre, deux d'entre elles sont différentes : dans l'exemplaire usé ( $\left.{ }^{\circ} 472\right)$, entre les pages 68 et 69, la colonne rostrale est complète avec les inscriptions du monument et I. Goerle del:, alors qu'elle est simplifiée dans la planche qui orne l'exemplaire en meilleur état ( $\left.\mathrm{n}^{\circ} 487\right)$; de même, entre les pages 70 et 71 du $\mathrm{n}^{\circ} 487$, la planche XXIV représentant la colonne Trajane, a une légende en latin: COLUMNA Imp. TRAJANI, in ipsius Foro, et comprend des détails de la base et du sommet que n'offre pas la planche incluse dans le $n^{\circ} 472$.

\section{RÉSUMÉS}

Résumé

Le repérage, à la bibliothèque de l'Académie des sciences de Saint-Pétersbourg, des guides de la Rome antique ayant fait partie de la bibliothèque de Pierre le Grand, laisse apparaître des lacunes troublantes. Des titres fondamentaux sont absents, ce qui peut être dû à de multiples facteurs. En revanche, trois ouvrages importants ont été retrouvés, dont le plus notable est Beschryving van Oud en Nieuw Rome de François Deseine, in-folio publié à Amsterdam en 1704 et orné de superbes planches. Deux d'entre elles représentent, de façon théâtrale et sans rapport avec la réalité, la colonne Antonine et la colonne Trajane vues à travers un arc de triomphe, ce qui a pu constituer une source pour la mise en scène par Karl Ivanovič Rossi de la colonne Alexandre érigée en 1834 devant le Palais d'Hiver.

Guides of ancient Rome from Peter the Great's library

When trying to locate the guides of ancient Rome that had been part of Peter the Great's library in the collection of the Academy of Sciences in Saint-Petersburg, one encounters some puzzling gaps. Basic titles are absent, which may be due to many reasons. On the other hand, three major works have been retrieved, the most important being François Deseine's Beschryving van Oud en Nieuw Rome, a folio published in Amsterdam in 1704 and adorned with splendid plates. Two of these plates represent, in a theatrical way by no means true to reality, the Antonine and Trajan Columns seen through a triumphal arch. This setting may have inspired Karl Ivanovich Rossi's mise en scene of Alexander's Column in front of the Winter Palace.

\section{AUTEUR}

\section{PHILIPPE SÉNÉCHAL}

Université de Picardie Jules Verne 